\title{
Survey Analysis of Robust and Real-Time Multi-Lane and Single Lane Detection in Indian Highway Scenarios
}

\author{
Dr. A. Sai Hanuman ${ }^{1, *}$ and G. Prasanna Kumar $^{2}$ \\ ${ }^{1}$ Professor, Computer Science and Engineering, GRIET, Hyderabad, Telangana, India. \\ ${ }^{2}$ Master of Technology, Computer Science and Engineering, GRIET, Hyderabad, Telangana, India.
}

\begin{abstract}
Studies on lane detection Lane identification methods, integration, and evaluation strategies square measure all examined. The system integration approaches for building a lot of strong detection systems are then evaluated and analyzed, taking into account the inherent limits of camera-based lane detecting systems. Present deep learning approaches to lane detection are inherently CNN's semantic segmentation network the results of the segmentation of the roadways and the segmentation of the lane markers are fused using a fusion method. By manipulating a huge number of frames from a continuous driving environment, we examine lane detection, and we propose a hybrid deep architecture that combines the convolution neural network $(\mathrm{CNN})$ and the continuous neural network $(\mathrm{CNN})(\mathrm{RNN})$. Because of the extensive information background and the high cost of camera equipment, a substantial number of existing results concentrate on vision-based lane recognition systems. Extensive tests on two large-scale datasets show that the planned technique outperforms rivals' lane detection strategies, particularly in challenging settings. A CNN block in particular isolates information from each frame before sending the $\mathrm{CNN}$ choices of several continuous frames with time-series qualities to the RNN block for feature learning and lane prediction.
\end{abstract}

\section{Introduction}

The demand for transport has increased enormously over the last two decades. There are now more automobiles on the road, which sadly means that traffic accidents are on the rise. [2] Significant efforts have been made to improve driver safety education and the construction of an accident prevention system. [3] Machine vision systems are crucial in today's cars for improved driver assistance systems with safety features. As a result, scientists and engineers have developed a range of technologies that use machine vision to enable the autonomy of intelligent vehicles, such as Lane Departure Warning (LDW), Adaptive Cruise Control (ACC), and Lane Center, among others. In recent years, machine vision research for road vehicles has progressed dramatically, and lane border identification has been a hot topic. The automatic lane detector should be able to recognize straight and curved lane boundaries, as well as different lane markers (single or double, solid or broken) and pavement edges [7]. Their reported uses are restricted to tiny images. As the image size grows larger, processing time and memory costs increase rapidly, and random aligned disturbances become audible,

*Corresponding author: prasannakumargourla@gmail.com attempts to apply HT to large-scale images are usually avoided. Engineering drawings, unfortunately, are frequently fairly large [8]. A visual apparatus that can differentiate numerous lanes on a highway is described in this paper. Initially, the system detected the center lane. On the assumption that the center lane markers were parallel to each other at the same interval and that the road was flat, the position of the adjacent lanes was determined in the top image by offsetting the central lane marks. [10].

Whereas a handmade choice produced by difficult way of fine standardization, the majority of lane recognition tactics utilize ancient laptop vision-based procedures. Such specialized alternatives operate in a controlled environment and do not appear to be powerful enough in complex driving scenarios, making them unsuitable for reasonable planning A laptop vision technique developed using $\mathrm{CNN}$ has the ability to offer lane detection resolution that is both dependable and accurate. The performance of recent lane detection methods that incorporated CNN language segmentation was outstanding. Despite their high performance, CNN-based segmentation algorithms require post-processing and model fitting to produce final lane predictions [4]. The deep 
convolution neural network (DCNN) is one of them, and it excels at image and video feature abstraction by analyzing input signals with multiple degrees of convolution. The deep recurrent neural network (DRNN) is good at data prediction for time-series signals because it recursively evaluates the signal by dividing it into sequential blocks and constructing full affiliation layers between them for status propagation. In terms of lane detection, the continuous driving scene photos appear to constitute time series that may be analyzed using DRNN.

\section{Methodology}

\subsection{Machine Learning-Based Lane Detection Algorithms:}

[2] Some studies focus on lane marking detective work utilizing modern machine learning, while others use traditional image processing-based methods to discover lane markings. [7] For a range of road surface types, lane configurations, weather conditions, and noise, the precision and robustness of road-bound detection for an autonomous vehicle is a difficult task. To overcome these concerns while keeping the high accuracy advantage of RHT, adaptive RHT (Randomized Hough transform) is used, this is an effective technique to apply RHT [7]. Pre-processing, post-processing, and road lane modeling are the three stages of the lane recognition approach [2]. This section delves into the details of these stages, as well as the calculations that accompany them.

\subsubsection{Pre-processing Stage}

Because Canny Edge Detection only works with monochromatic pictures, the image is also transformed to grey scale. Image processing techniques such as erosion, dilation, and image smoothing are used to remove noise.

\subsubsection{The post-processing phase}

After the data has been analyzed, canny edge detection is performed. The smooth image's stage recognition feature recognizes lines and edges.

\subsubsection{Road Lane Modeling}

The most likely left and right lane markers are calculated using computation. In this calculation, the line parameters rho(T) and theta(d) are used. You can achieve an average result by grouping comparable lines together.

\subsection{Deep Learning Techniques:}

Deep learning techniques have become one of the most popular academic topics in the last decade as a result of discoveries in deep network theories, parallel computing technologies, and large-scale data [1]. Many deep learning techniques demonstrate significant improvements in computer vision tasks when compared to standard methods, with dramatically increased detection and identification efficiency. The convolution neural network is one of the most extensively utilized approaches for object recognition research $(\mathrm{CNN})$. CNN has many advantages, including high detection precision, automated feature learning, and end-to-end recognition. Recently, some researchers were active in the application process.

The two types of evaluation processes are offline performance evaluation and online real-time trust evaluation. LSTM could be an important component in deep learning because of its capacity to analyze temporal data. Convolutional LSTMs (ConvLSTMs) are a sort of LSTM that is equipped with convolution operations and has been widely used in video analysis due to their feedback mechanism on temporal dynamics and abstraction power on picture illustration. ConvLSTM can be used in a variety of ways as a basic construction component. ConvLSTM's area unit is extensively utilized in linguistics video segmentation for efficiently processing two-dimensional time-series data. The video segmentation problem was turned into a regression problem by using a ConvLSTM between the convolutional encoder and decoder to anticipate each frame.

\subsection{Image Processing Techniques:}

[2] For detecting left and right lane markers, this study provides a robust road lane marker identification technique. Canny edge detection and Hough Transform optimization are used in the method. Images from a front-facing vision sensor positioned behind the windscreen and facing the lane are used as data by the device.

The left and right lane limitations are correctly shown on the original photo as a result of this technique. The Hough Transform yields a sequence of lines that characterize the situation as lane borders on the left and right. The average creates only one result by grouping all of the left lane boundaries into one category and all of the right lane boundaries into another.

[3]The Fuzzy Noise Reduction Filter (FNRF) is the first sub-algorithm, and it removes noise and smooth's down image sequences recorded by the camera. FNRF is used to increase the ROI's contrast level, which can help in lane detection. On a clear road, FNRF is 
detected 99.2 percent of the time, but only 92.1 percent of the time on a dark road. The FNFR generates positive results by reducing noise and increasing detection rates by an average of 13.30 percent.

\subsection{Convolution Neural Networks Techniques:}

For autonomous driving, accurate lane identification is a must-have feature. For crucial roles like overtaking assistants and route planning, ego and side lane positional awareness are also necessary. We describe how to use a CNN-based regression technique to detect several lanes and classify them based on their position in this study. Our regression technique removes the significant constraint of precisely categorizing each pixel from CNN segmentation-based algorithms that produce dense pixel masks.

In high contrast photos, UNet $(64.5 \%)$ and Lane Net $(65.7 \%)$, in particular, struggle to distinguish lane boundaries for mean intersection over union (MIOU). When the results of road and lane marker instance segmentation are combined, an area of ego-way, left and right adjacent lane is expected [5].

The lane's outcome will be represented as a collection of pixels.

When it comes to road segmentation, we can get consistent results. Multi-lane detection can be done by combining these two results. This technique appears to operate for a number of road settings, including unstructured roads with no markings, structured roads, and semi-structured roads with one to four lane lines, according to many testing.

In addition, the results of the multi-task network's recognition of road and lane markings are cutting-edge. To obtain a quantitative evaluation result, an annotation of the lane class (ego-lane ( 88 percent), left (78 percent), and right adjacent lane (76 percent) is added to the test photos.

The creation of the unique "video-based lane estimating and tracking" (VioLET) system [6] is motivated by these driver-assistance goals We'll examine the current state of the art in track position recognition and tracking, as well as compare and contrast techniques.

Large surveys of intelligent vehicles have looked at many of the lane position sensing techniques. While these publications are important for broad overviews of vision research for intelligent vehicles, they are only as detailed as they can be provided. By enabling the left and right boundaries to be merged, the assumption that the width of the road or lane is locally constant can considerably enhance performance.

One is the deep convolutional neural network (DCNN), which processes input data using many levels of convolution and excels at feature abstraction for images and videos.
The deep continuous neural network (DRNN) is capable of information prediction for time-series signals by recursively processing the signal by dividing it into sequential blocks and establishing full affiliation layers between them for status propagation. In terms of lane detection, continuous driving scene images appear to be a time series that may be handled using DRNN.

\section{Flow chart for lane detection}

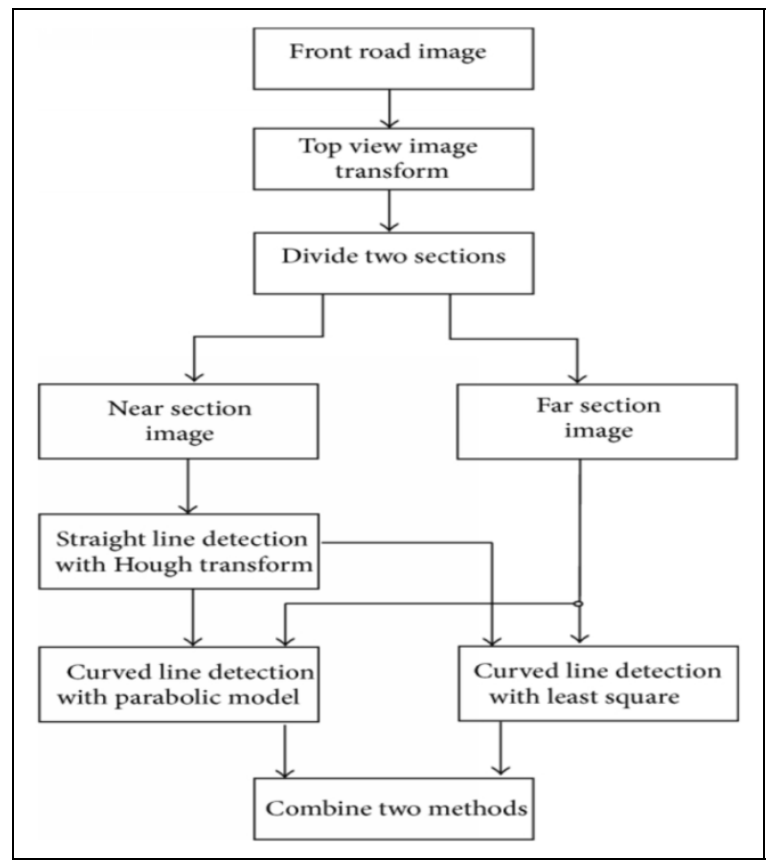

Fig. 1. Flow chart

\section{Comparison}

Table 1. Comparison of the methods used

\begin{tabular}{|c|c|c|c|}
\hline Methods & Algorithms & Accuracy & Reference \\
\hline $\begin{array}{c}\text { Vision } \\
\text { based } \\
\text { lane } \\
\text { detection } \\
\text {, Image } \\
\text { processin } \\
\mathrm{g} \\
\end{array}$ & $\begin{array}{c}\mathrm{CNN}, \\
\text { Hough } \\
\text { transform }\end{array}$ & $90 \%$ & $\begin{array}{c}\text { Reference } \\
-[1]\end{array}$ \\
\hline $\begin{array}{c}\text { Image } \\
\text { processin } \\
\mathrm{g}\end{array}$ & $\begin{array}{l}\text { Canny edge } \\
\text { detection }\end{array}$ & $91.2 \%$ & $\begin{array}{c}\text { Reference } \\
-[2]\end{array}$ \\
\hline $\begin{array}{c}\text { Fuzzy } \\
\text { Noise } \\
\text { Reductio } \\
\text { n Filter } \\
\text { (FNRF) }\end{array}$ & $\begin{array}{c}\text { Edge } \\
\text { detection }\end{array}$ & $87 \%$ & $\begin{array}{c}\text { Reference } \\
-[3]\end{array}$ \\
\hline $\begin{array}{c}\text { Multilan } \\
\mathrm{e}\end{array}$ & $\mathrm{CNN}$ & $75 \%$ & $\begin{array}{c}\text { Reference } \\
-[4]\end{array}$ \\
\hline
\end{tabular}




\begin{tabular}{|c|c|c|c|}
\hline detection & & & \\
\hline $\begin{array}{c}\text { Lane } \\
\text { Marking } \\
\text { detection }\end{array}$ & RANSAC & $\begin{array}{c}\text { Ego } \\
\text { lane } \\
88.02 \% \\
\text { Right } \\
\text { lane } \\
76.28 \\
\% \\
\text { Left lane } \\
78.60 \%\end{array}$ & $\begin{array}{c}\text { Reference } \\
-[5]\end{array}$ \\
\hline $\begin{array}{c}\text { Vide } \\
\text { o } \\
\text { base } \\
\text { dlane } \\
\text { detec } \\
\text { tion } \\
\text { and } \\
\text { tracki } \\
\text { ng } \\
\text { (VIOLE } \\
\text { T) } \\
\end{array}$ & $\begin{array}{l}\text { FNRF, } \\
\text { CNN, and } \\
\text { KNN }\end{array}$ & $92 \%$ & $\begin{array}{c}\text { Reference } \\
-[6]\end{array}$ \\
\hline $\begin{array}{c}\text { Machine } \\
\text { learning } \\
(\mathrm{ML})\end{array}$ & $\begin{array}{c}\text { Randomized } \\
\text { Hough } \\
\text { transform } \\
\end{array}$ & $82 \%$ & $\begin{array}{c}\text { Reference- } \\
{[7]}\end{array}$ \\
\hline $\begin{array}{l}\text { Line } \\
\text { Reco } \\
\text { gniti } \\
\text { on } \\
\text { meth } \\
\text { od }\end{array}$ & $\begin{array}{l}\text { Hough } \\
\text { transform }\end{array}$ & $82 \%$ & $\begin{array}{c}\text { Reference- } \\
{[8]}\end{array}$ \\
\hline $\begin{array}{c}\text { CNN-LD } \\
\text { baselane } \\
\text { detection } \\
\text { ML }\end{array}$ & $\begin{array}{c}\mathrm{CNN}, \\
\text { Hough } \\
\text { transform }\end{array}$ & $96 \%$ & $\begin{array}{c}\text { Reference- } \\
{[9]}\end{array}$ \\
\hline GPS & HT -ROI & $70 \%$ & $\begin{array}{c}\text { Reference- } \\
{[10]}\end{array}$ \\
\hline
\end{tabular}

\section{Inferences}

So, by comparing all the methods and algorithms CNN is an environment friendly cognizance algorithm which is widely used in sample focus and image processing. It has many elements such as simple structure, less education parameters and adaptability. It has become a warm subject matter in voice analysis and image recognition.

Hough transform can be used to realize lines and circles or different parametric curves [1].

Canny edge detection: the canny edge detector is an part detection operator that uses a multistage algorithm to notice a huge vary of edge in photographs [2].

FNRF: Fuzzy filter is presented for the noise discount of pix corrupted with adaptive noise [3].

The RANSAC algorithm is a learning method to estimate parameters of a model via random sampling of observed data [5].

KNN It makes use of data with countless training to predict the classification of the new sample point [6].
RHT is specific from HT in that it tries to keep away from conducting the computationally pricey vote casting process for each nonzero pixel in the photo by means of taking benefit of the geometric houses of analytical curves, and accordingly improve the time effectively and reduce the storage requirement of the authentic algorithm [7].

CNN, KNN, and RANSAC these are the most used algorithms in previous projects but $\mathrm{KNN}$ with large data the prediction stage might be slow, $\mathrm{CNN}$ has several layers so that the training process takes a lot of time if the system does not consist of a good GPU and RANSAC there are no upper bonds on the time it takes to compute any parameters and it computes limit number of solutions whereas Hough transform and canny edge detection algorithms are giving good accuracy as per my survey and these algorithms can detect curves and edges in single and multilane clearly. So these are the algorithms to consider for my project can be used more practically.

\section{Conclusion}

In this survey it concludes that it gives the qualified analysis of the Robust and real-time multi-lane and single lane detection in Indian highway scenarios. Different types of lane detection are done based on different types of vehicles and road as in moving vehicles or video based. In each paper they are used different datasets and algorithms for the efficient accuracy. For more efficiency there must be well applied algorithm which must be correctly implemented along with the consider dataset. According to survey analysis Hough transform and canny edge detection are the best resulting algorithms.

\section{References}

1. Y. Xing, C. Lv, L. Chen, H. Wang, H. Wang, D. Cao, E. Velenis, and F.-Y. Wang, IEEE/CAA Journal of Automatica Sinica 5, 645 (2018)

2. C. Y. Low, H. Zamzuri, and S. A. Mazlan, in 2014 5th International Conference on Intelligent and Advanced Systems (ICIAS), pp. 1-4 (2014)

3. H. Bilal, B. Yin, J. Khan, L. Wang, J. Zhang, and A. Kumar, in 2019 Chinese Control Conference (CCC), pp. 6772-6777 (2019)

4. S. Chougule, N. Koznek, A. Ismail, G. Adam, V. Narayan, and M. Schulze, in Proceedings of the European Conference on Computer Vision (ECCV) Workshops (2018)

5. P. Liu, M. Yang, C. Wang, and B. Wang, in 2018 Chinese Automation Congress (CAC), pp. 27502755 (2018) 
6. J. C. McCall and M. M. Trivedi, IEEE Trans. Intell. Transp. Syst. 7, 20 (2006)

7. Q. Li, N. Zheng, and H. Cheng, IEEE Trans. Intell. Transp. Syst. 5, 300 (2004)

8. J. Song and M. R. Lyu, Pattern Recognit. 38, 539 (2005)

9. S. K. Satti, K. Suganya Devi, P. Dhar, and P. Srinivasan, ICT Express 7, 99 (2021)

10. Y. Jiang, F. Gao, and G. Xu, in 2010 International Conference on Image Analysis and Signal Processing, pp. 114-117 (2010) 